\title{
What is timed in a fixed-interval temporal bisection procedure?
}

\author{
Adam E. Fox ${ }^{1}$ Katelyn E. Prue ${ }^{1}$ Elizabeth G. E. Kyonka ${ }^{2}$
}

Published online: 29 April 2016

(C) Psychonomic Society, Inc. 2016

\begin{abstract}
Recent research on interval timing in the behavioral and neurological sciences has employed a concurrent fixedinterval (FI) procedure first reported by Platt and Davis (Journal of Experimental Psychology: Animal Behavior Processes, 9, 160-170, 1983). Studies employing the task typically assess just 1 dependent variable, the switch/bisection point; however, multiple measures of timing are available in the procedure and it is unclear (a) what is timed (i.e., learned) by subjects and (b) what other measures might tell us about timing in the task and generally. The main objective of the current experiment was to utilize multiple dependent measures of timing accuracy and precision derived from the task to assess whether the 2 FIs are timed independently or if timing 1 FI interferes with timing the other, and vice versa. Four pigeons were exposed to an FI temporal bisection procedure with parametric manipulations across two phases. In the constant phase, the short FI was always the same; the long FI was 2 to 16 times the short FI and changed across conditions. In the proportional phase, the long FI was always 4 times the duration of the short FI. Across both phases, pigeon mean bisection points were near the geometric mean of the 2 FIs.
\end{abstract}

Data were collected at West Virginia University with funding from the Department of Psychology. Portions of these data were presented at the Society for the Quantitative Analysis of Behavior Conference in 2014. K. E. P. now at Salem State University.

Adam E. Fox

afox@stlawu.edu

Elizabeth G. E. Kyonka

ekyonka@une.edu.au

1 Department of Psychology, St. Lawrence University, Canton, NY 13617, USA

2 Department of Psychology, University of New England, Armidale, NSW 2351, Australia
Coefficients of variance increased as the durations to be timed increased. Results suggested pigeons' timing of the short FI was affected by the presence of the long FI, and vice versa. The FI temporal bisection task offers multiple dependent variables for analysis and is well suited for studying temporal learning and decision making.

Keywords Interval timing - Temporal bisection $\cdot$ Scalar invariance $\cdot$ Response gradient $\cdot$ Pigeons $\cdot$ Key peck

The ability to discriminate and categorize time, and to respond appropriately to intervals of different durations, is a critical component of communication and survival for nonhumans and humans alike. Temporal judgment is as essential for an animal estimating whether it can outrun a predator as for a driver determining when to change lanes on a highway. Furthermore, organisms are often required to time multiple intervals simultaneously. For example, in a social interaction, a human might simultaneously estimate the time since the start of the interaction, the time since their last verbal and nonverbal behaviors, and the time since the last verbal and nonverbal behaviors of others. One way temporal discrimination of multiple intervals is studied is by using discrete-trial temporal bisection procedures (e.g., Church \& Deluty, 1977; Cordes \& Meck, 2014; Machado \& Pata, 2005; Pinheiro de Carvalho, Machado, \& Tonneau, 2016). First, the subject is trained to make one response when a stimulus is presented for a short duration and another response when it is presented for a long duration. Next, a range of durations are presented repeatedly; the task is to classify the duration as short or long by making the appropriate response. The resultant psychophysical function (i.e., the proportion of trials in which the subject makes the long response plotted as a function of stimulus duration) is typically ogival in shape. 
Platt and Davis (1983) reported results of an alternative temporal bisection task in which two fixed interval (FI) schedules were presented concurrently. The organism is required to time two intervals simultaneously instead of classifying a single interval as in the discrete-trial version of the task described above. In the Platt and Davis procedure, one FI was a relatively short duration (e.g., $2 \mathrm{~s}$ ) and the other was a relatively long duration (e.g., $8 \mathrm{~s}$ ). At the start of a trial, both FI options were presented. On half of trials, a reinforcer was available for pecking one key after the short interval. On the other half of trials, a reinforcer was available for pecking the other key after the long interval. On trials in which a reinforcer was scheduled on the long-interval/location, pigeons typically pecked the short key at the beginning of the interval, then switched to the long key after the short FI had elapsed. The time in a trial at which there was an equal probability of responding on either FI (the bisection point) was near the geometric mean of the two FI durations. Plotting the proportion of long responses as a function of time since trial onset produced a psychophysical function similar to the function observed in discrete-trial bisection tasks.

Platt and Davis's (1983) FI temporal bisection task offers some advantages over other timing tasks, including the peak procedure (Catania, 1970; Roberts, 1981; Sargisson, Lockhart, McEwan, \& Bizo, 2016; Wilson, Matell, \& Crystal, 2015) and the discrete-trial version of the temporal bisection task (e.g., Church \& Deluty, 1977). First, it allows assessment of timing of multiple intervals simultaneously, which likely better represents real-world scenarios. Second, it requires very little, if any, pretraining. As soon as a subject has been trained to respond, testing can begin. Both the peak interval procedure and the discrete-trial temporal bisection task may require considerable training, depending on the research goals. Third, the FI temporal bisection task makes reinforcement available on all trials. In the peak interval procedure, the discrete-trial temporal bisection task, and on the concurrent VI VI free-operant psychophysical choice task (e.g., Cheung et al., 2007), reinforcers are typically withheld on test trials. Withholding reinforcers on test trials may disrupt or systematically affect relevant dependent measures (Staddon \& Innis, 1969). Finally, the FI temporal bisection task produces multiple timing-dependent measures (discussed in detail below), allowing for more analyses and, potentially, a more detailed picture of how independent variables might affect timing accuracy and precision systematically.

Perhaps for these and other reasons, Platt and Davis's (1983) FI temporal bisection procedure is gaining popularity in the behavioral and neurological timing literature (Balci, Freeston, \& Gallistel, 2009; Balci et al., 2008; Daniels, Fox, Kyonka, \& Sanabria et al. 2015a; Daniels, Watterson, et al., 2015b; Gallistel et al., 2014; Maggi et al., 2014; Tosun, Gur, \& Balci, 2016); however, much is not yet understood about timing in the task. Previous experiments generally have not been designed to characterize temporal learning in the task by assessing responding at different pairs of short-long intervals. Instead, the research objectives have been to evaluate effects of pharmacological, genetic, or environmental manipulations unrelated to the durations of short and long FIs. These studies have focused nearly exclusively on a single dependent measure: the bisection point (or latency to switch to the long FI on trials in which the reinforcer is available at that location). Other aspects of responding in this task, including response gradients for the short FI and long FI schedules, presumably come under temporal control. However, it is not entirely clear from bisection points alone what is timed (i.e., learned) by the subject. For instance, responding throughout each long trial could be determined by the short FI, the long FI, the difference between short and long, the short:long ratio, or a combination of these or other factors.

A more complete understanding of the temporal determinants of behavior and the utility of multiple dependent variables in the procedure would expand its utility as a timing task and provide insight into timing processes more generally. This was the main aim of the present experiment. The Platt and Davis (1983) procedure yields at least four important measures of timing. First, the response gradient for responding on the long FI key should approximate response gradients from traditional FI schedules. Generally, response gradients should be ogival, with a midpoint (time in which responding reaches half its maximum rate) at approximately two-thirds of the interval (Schneider, 1969) and a slope that flattens in proportion to increases in FI duration.

Second, the response gradient for the short FI key should approximate a peak distribution similar to those observed in peak procedures (Catania, 1970; Roberts, 1981). In their simplest form, peak procedures expose organisms to traditional FI schedules and occasionally "peak" trials (trials in which food is never delivered) are inserted-response gradients from these trials approximate a Gaussian function, with a peak location at the time of the FI and a standard deviation that increases proportionally with the interval to be timed.

Third, when responding on long trials is aggregated and plotted as a proportion of responding on the long FI, the gradient should have approximately the same shape as psychophysical functions obtained from free-operant psychophysical choice procedures (Stubbs, 1980; Bizo \& White, 1994, 1995) and the Church and Deluty (1977) discrete-trial version of the temporal bisection task. In the Platt and Davis (1983) task, the centers of these functions represent the mean switch point from the short to the long FI. This measure should approximate the bisection point obtained using discrete-trial style temporal bisection tasks - that is, it should occur near the geometric mean (Fetterman \& Killeen, 1995; Platt \& Davis, 1983).

Fourth, if scalar variance is observed, coefficients of variation for temporally controlled behavior should be constant. 
For response gradients, response variability should be proportional to the time of the mean response. For example, the slopes of the psychophysical functions represent a measure of timing precision - steeper slopes indicate more consistency in the time in which the organism switched from the short to the long FI. Psychophysical function slopes should flatten proportionally as the FI durations increase.

Scalar variance is an established feature of timing behavior: as the duration to be timed increases, variability in timing of responses increases proportionally. Scalar variance predicts that if variability in timing behavior is normalized with respect to the target time, then a constant fraction should be observed across durations. To do this, a Weber fraction is often calculated. However, Platt and Davis (1983) did not report measures of variability for timing behavior on the task. In the their experiments, the slope of the ogival psychophysical function when the data are plotted as a proportion of long FI responses, divided by the bisection point of the same ogive function, yields an index of normalized timing precision - a coefficient of variation $(\mathrm{CV})$. If scalar variance was observed in their procedure, CVs should be roughly constant across FI durations. Likewise, the same should be true for ogive slopes divided by midpoints for responding on the long FI and for Gaussian standard deviations divided by peak times for responding on the short FI. In Fetterman and Killeen's (1995) similar categorical scaling task, scalar variance was observed: when response gradients were normalized across different FIs, they were similar. However, in a task similar to the one used presently, Balci et al. (2008) reported increasing $\mathrm{CVs}$ with increasing FIs - a violation of scalar variance.

In the present study, pigeons were exposed to a procedure similar to that used by Platt and Davis (1983). In one phase of the experiment, the short and long FIs changed proportionally; in a second phase the short FI was always $2 \mathrm{~s}$ and the long FI changed.

Our first objective was to obtain bisection points and measures of timing variability across multiple FI duration pairsgenerally a replication of previous work; however, neither Platt and Davis (1983) nor researchers since have provided measures of standardized variability (CVs) in the task in this way (but cf. Balci et al., 2008). The second objective was to highlight the potential utility of multiple dependent measures in the task, compare how they might be similar to dependent measures from other timing tasks, and assess how they are affected by manipulations of FI duration. In doing so, we hoped to shed light on the question of what is timed in the task and how. For example, changing both FI durations may affect timing in all dependent variables, but changing one FI duration may affect only some, or may affect dependent measures differently. One hypothesis is that temporal control is simultaneous and independent: the short FI determines responding on the short FI option, the long FI determines responding on the long FI option, and that they do not interfere. Simultaneous, independent timing would predict scalar variance in response gradients in addition to bisection at the geometric mean. However, simultaneous timing of the two FIs may result in interference, perhaps from the ratio of or difference between the short and long FIs. If timing of the two FIs interferes with one another, scalar variance might be observed in some circumstances but not others, and dependent measures may be affected differently, depending on the manipulation of FI durations. This question of timing independence or interference is important in relation to the future utility of the FI temporal bisection task and from a timing theory perspective in terms of how organisms time multiple intervals simultaneously.

\section{Method}

Subjects Four experienced White Carneau Pigeons, numbered 501 to 504 , were maintained at $85 \%$ of their freefeeding weight plus or minus $15 \mathrm{~g}$ through appropriate postsession feedings. Pigeons were housed individually in a vivarium with a 12-hr light/12-hr dark cycle and had continuous access to water. All pigeons had previous experience pecking keys for access to food.

Apparatus Standard operant chambers $(25.5 \mathrm{~cm}$ deep $\times 32 \mathrm{~cm}$ wide $\times 33.5 \mathrm{~cm}$ high) were enclosed in sound-attenuating boxes equipped with ventilation fans. Each chamber contained three response keys arranged $6 \mathrm{~cm}$ apart and $24 \mathrm{~cm}$ above the floor of the chamber. Response keys could be illuminated red, green, or white. A grain hopper $(5.5 \mathrm{~cm}$ high $\times 6 \mathrm{~cm}$ wide) was located below the middle response key and $5.5 \mathrm{~cm}$ from the floor. A house light was located at the top of the chamber on the wall opposite of the response keys. The grain hopper aperture was illuminated during reinforcer presentation, and the hopper contained Purina Nutrigrain pigeon pellets. A force of approximately $0.15 \mathrm{~N}$ was required to register a response on any key. All experimental events were controlled through a computer and MED-PC interface located in an adjacent room.

Procedure At the start of each trial, the left and right keys were lighted white. The left key was always associated with a relatively short FI and the right key with a relatively long FI. There were two types of trials that occurred: short and long, but both keys were concurrently available for the duration of both trial types. On short trials, food was delivered contingent on a key peck to the left key after the short interval elapsed. On long trials, food was delivered contingent on a key peck to the right key after the long interval elapsed. Food reinforcement consisted of three, 2.5-s grain hopper presentations delivered consecutively, separated by $0.5 \mathrm{~s}$. The next trial followed immediately after reinforcement. A 3-s limited hold was in place on both keys - a reinforcer could only be earned for a key peck within $3 \mathrm{~s}$ of either FI elapsing. If a reinforcer was not 
collected because the pigeon did not peck the appropriate key within $3 \mathrm{~s}$ after the FI elapsed, a 10-s blackout occurred during which all the lights in the chamber were extinguished, followed by the next trial. This contingency was included to ensure that pigeons pecked both keys and that sessions ended within a reasonable amount of time. Short and long trials were not differentially signaled in any way except by the passage of time. Each session consisted of 36 short and 36 long trials. Trial type was determined by random selection without replacement from blocks of six short and six long trials.

Pigeons were exposed to two phases in counterbalanced order: constant and proportional. In the constant phase, the short FI was always $2 \mathrm{~s}$ and the long FI was $4 \mathrm{~s}, 8 \mathrm{~s}, 16 \mathrm{~s}$, or $32 \mathrm{~s}$ across separate conditions. In the proportional phase, the short and long FI varied proportionally: 1 and $4 \mathrm{~s}, 2$ and $8 \mathrm{~s}, 4$ and $16 \mathrm{~s}$, and 8 and $32 \mathrm{~s}$ across conditions. In each phase, pigeons were exposed to the 2 and $8 \mathrm{~s}$ condition first, followed by the remaining conditions in a randomly determined order, including a replication of the 2 and $8 \mathrm{~s}$ condition with the constraint that it could not occur immediately following the first 2 and $8 \mathrm{~s}$ condition. Table 1 shows the order of conditions and number of sessions in each condition in parentheses for each pigeon. Conditions lasted a minimum of seven sessions (with the exception of the first condition, which lasted a minimum of 21 sessions) and, until mean latency to peck the long FI key on long trials, showed no visual trend across the last five sessions. In nearly all cases, behavior stabilized in the minimum seven sessions. After the first condition, the number of sessions in each condition exceeded 10 only once across pigeons. Pigeons always completed all 72 trials in less than 70 minutes. Sessions were conducted 7 days a week. Data from long trials in the last three sessions of each condition were used in analyses.

\section{Results}

Figure 1 shows group mean response gradients in all four conditions from each phase. For simplicity, the data from the 2 vs $8 \mathrm{~s}$ conditions were combined in both phases because behavior was not systematically different in the two exposures. Trials were divided into 0.1 -s bins. $X$-axes (time) were normalized to the short FI duration (i.e., the short FI $=1$ ). We calculated short-key and long-key responses per minute for each bin. Pigeons pecked on the short FI key at the start of long trials and the long FI key at the end of long trials. Local response rate on the short key peaked around the short FI duration, with the 1 vs. $4 \mathrm{~s}$ condition as an exception. Response rate on the long FI key increased as long trials progressed.

Psychophysical functions Short and long response gradients were compared to obtain the proportion of responding on the long FI as a function of time for each pigeon in every condition. For each 0.1-s bin, we calculated the proportion of long responses as long-key pecks/(short-key pecks + long-key pecks). This yielded ogival psychophysical functions. For a quantitative characterization of the functional relation between the proportion of responding occurring on the long FI and time since the start of a trial, an ogive function (Guilhardi $\&$ Church, 2005) was fitted to the response proportion gradients using Eq. 1:

$y(t)=\frac{1}{1+e^{-(t-a) / b}}$

In Eq. 1, the $a$ parameter determined the center of the function. For a psychophysical function, it is the bisection point,

Table 1 Order of conditions

\begin{tabular}{|c|c|c|c|c|c|c|c|c|c|c|c|c|}
\hline \multirow[t]{3}{*}{ Condition } & \multicolumn{12}{|l|}{ Pigeon } \\
\hline & \multicolumn{3}{|l|}{501} & \multicolumn{3}{|l|}{502} & \multicolumn{3}{|l|}{503} & \multicolumn{3}{|l|}{504} \\
\hline & Short FI & Long FI & & Short FI & Long FI & & Short FI & Long FI & & Short FI & Long FI & \\
\hline 1 & 2 & 8 & (21) & 2 & 8 & (21) & 2 & 8 & (21) & 2 & 8 & (21) \\
\hline 2 & 4 & 16 & (7) & 8 & 32 & (7) & 2 & 32 & (7) & 2 & 4 & (7) \\
\hline 3 & 8 & 32 & (7) & 1 & 4 & (7) & 2 & 4 & (7) & 2 & 16 & (7) \\
\hline 4 & 1 & 4 & $(9)$ & 2 & 8 & $(8)$ & 2 & 16 & $(8)$ & 2 & 8 & (7) \\
\hline 5 & 2 & 8 & (7) & 4 & 16 & (7) & 2 & 8 & (7) & 2 & 32 & (7) \\
\hline 6 & 2 & 32 & $(9)$ & 2 & 8 & (10) & 4 & 16 & (10) & 2 & 8 & (7) \\
\hline 7 & 2 & 8 & $(7)$ & 2 & 4 & (7) & 8 & 32 & $(7)$ & 4 & 16 & (7) \\
\hline 8 & 2 & 4 & $(8)$ & 2 & 16 & $(8)$ & 2 & 8 & $(8)$ & 8 & 32 & (9) \\
\hline 9 & 2 & 16 & (7) & 2 & 8 & (7) & 1 & 4 & (7) & 1 & 4 & (7) \\
\hline 10 & 2 & 8 & (7) & 2 & 32 & (14) & 2 & 8 & (7) & 2 & 8 & (7) \\
\hline
\end{tabular}

Note. All FIs shown in seconds. The number of sessions for each condition shown in parentheses 

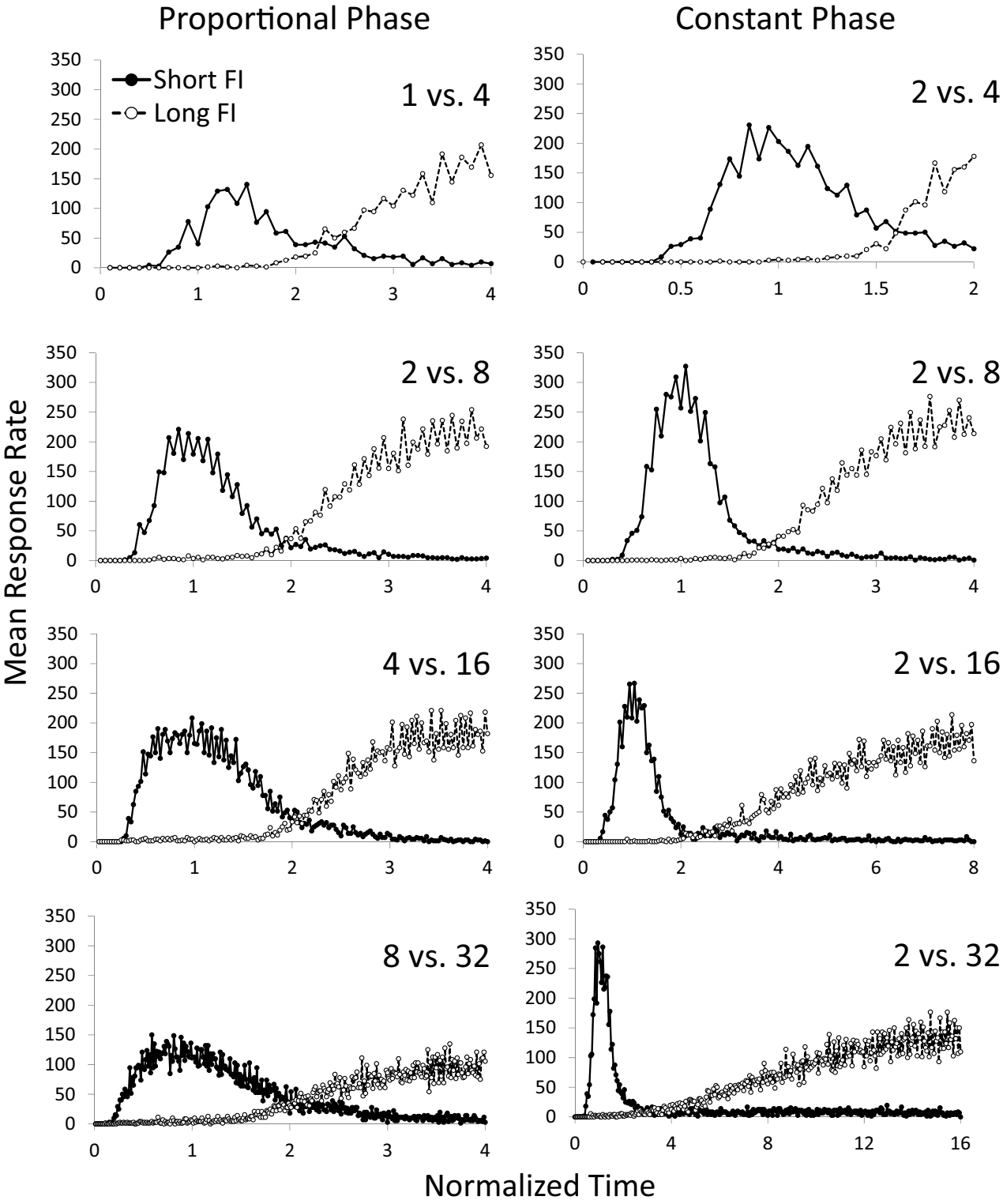

Fig. 1 Mean response gradients for pigeons across conditions and phases. $X$-axes are normalized to the duration of the short FI (i.e., short FI duration $=1.0$ ) Throughout figure, for consistency, please change "v" to "vs." as in the text (i.e., 1 vs. 4).New figure attached.

the time when response rate on the short and long FIs was equal. The $b$ parameter determined the slope of the function. Smaller estimates of $b$ indicate steeper slopes and therefore less variability in the time when the switch from the short to the long FI occurred across trials. Table 2 shows parameters obtained from fitting Eq. 1 to psychophysical functions for each pigeon in each condition. All psychophysical functions were well described by Eq. 1: Mean VAC across pigeons and conditions was .94 ( $S D=.05$, Range: 0.76-0.99).

Figure 2 shows group mean psychophysical functions as a function of normalized time (i.e., the long FI duration $=1$ ) in the proportional and the constant phases. For simplicity, data from the 2 vs. $8 \mathrm{~s}$ conditions of both phases were combined. The gradients confirm that responding shifted from near exclusivity on the short FI key at the beginning of a trial to near exclusivity on the long FI key by the end of a trial.

The slope (b) parameters for each pigeon in each condition were entered into a $2 \times 4$ repeated-measures analysis of variance (ANOVA), with phase and long FI duration as factors. There was a main effect of long FI duration, $F(3,9)=16.64, p<.001$, $\eta_{\mathrm{p}}{ }^{2}=.85$, but no main effect of phase $F(1,3)=0.05, p=.84$, $\eta_{\mathrm{p}}{ }^{2}=.02$, or Phase $\times$ Long FI interaction, $F(3,9)=0.32, p=.81$, $\eta_{\mathrm{p}}{ }^{2}=.10$. In both phases, functions for individual pigeons were flattest (with the largest $b$ parameters) in conditions with 32-s long FI schedules. When $x$-axes were normalized relative to long FI duration, mean $b$ was 0.10 (Range: $0.09-0.12$ ) in the $8 \mathrm{vs} .32 \mathrm{~s}$ condition of the proportional phase and .09 (Range: $0.04-0.16)$ in the 2 vs. 32 condition of the constant phase. For 
Table 2 Ogive model parameter estimates (Eq. 1) for psychophysical functions In the table note, please explain the meaning of parenthetical (1) and (2).We added a statement describing the meaning of (1) and (2) in the table headings, thanks.

\begin{tabular}{|c|c|c|c|c|c|c|c|c|c|c|c|}
\hline \multirow[t]{2}{*}{ Pigeon } & \multirow[t]{2}{*}{ Parameter } & \multicolumn{5}{|c|}{ Proportional Phase } & \multicolumn{5}{|c|}{ Constant Phase } \\
\hline & & 1 vs. 4 & 2 vs. 8 (1) & 2 vs. 8 (2) & 4 vs. 16 & 8 vs. 32 & 2 vs. 4 & 2 vs. $8(1)$ & 2 vs. 8 (2) & 2 vs. 16 & 2 vs. 32 \\
\hline \multirow[t]{4}{*}{501} & Center $(a)$ & 2.05 & 3.40 & 4.60 & 7.80 & 15.80 & 3.01 & 3.59 & 3.82 & 4.40 & 7.49 \\
\hline & Slope $(b)$ & 0.22 & 0.31 & 0.34 & 0.51 & 2.04 & 0.22 & 0.16 & 0.11 & 0.66 & 1.51 \\
\hline & VAC & 0.98 & 0.98 & 0.99 & 0.94 & 0.92 & 0.98 & 0.99 & 0.98 & 0.92 & 0.82 \\
\hline & $\mathrm{CV}$ & 0.11 & 0.09 & 0.07 & 0.07 & 0.13 & 0.07 & 0.04 & 0.03 & 0.15 & 0.20 \\
\hline \multirow[t]{4}{*}{502} & Center $(a)$ & 2.50 & 4.03 & 4.11 & 9.13 & 15.30 & 3.03 & 3.32 & 4.11 & 5.64 & 6.49 \\
\hline & Slope $(b)$ & 0.27 & 0.15 & 0.48 & 0.78 & 2.14 & 0.20 & 0.09 & 0.23 & 0.98 & 1.35 \\
\hline & VAC & 0.96 & 0.98 & 0.94 & 0.93 & 0.92 & 0.99 & 0.97 & 0.98 & 0.93 & 0.88 \\
\hline & $\mathrm{CV}$ & 0.11 & 0.04 & 0.12 & 0.09 & 0.14 & 0.07 & 0.03 & 0.06 & 0.17 & 0.21 \\
\hline \multirow[t]{4}{*}{503} & Center $(a)$ & 2.15 & 4.43 & 3.86 & 8.22 & 18.01 & 3.40 & 4.64 & 3.33 & 5.30 & 5.62 \\
\hline & Slope $(b)$ & 0.16 & 0.49 & 0.44 & 1.29 & 2.87 & 0.28 & 0.39 & 0.10 & 1.07 & 0.94 \\
\hline & VAC & 0.99 & 0.97 & 0.97 & 0.94 & 0.96 & 0.95 & 0.98 & 0.99 & 0.89 & 0.80 \\
\hline & $\mathrm{CV}$ & 0.07 & 0.11 & 0.11 & 0.16 & 0.16 & 0.08 & 0.08 & 0.03 & 0.20 & 0.17 \\
\hline \multirow[t]{4}{*}{504} & Center $(a)$ & 3.11 & 3.61 & 3.98 & 9.11 & 17.77 & 3.14 & 4.03 & 4.58 & 5.16 & 7.92 \\
\hline & Slope $(b)$ & 0.34 & 0.40 & 0.66 & 1.14 & 3.61 & 0.19 & 0.47 & 0.62 & 0.92 & 2.48 \\
\hline & VAC & 0.92 & 0.92 & 0.93 & 0.96 & 0.92 & 0.98 & 0.96 & 0.93 & 0.98 & 0.76 \\
\hline & $\mathrm{CV}$ & 0.11 & 0.11 & 0.17 & 0.13 & 0.20 & 0.06 & 0.12 & 0.14 & 0.18 & 0.31 \\
\hline
\end{tabular}

Note . VAC $=$ variance accounted for; $\mathrm{CV}=$ coefficient of variation. Paranthetical (1) and (2) in headings respectively refer to the initial 2 vs. 8 condition in a phase and the replication

all other conditions, $b$ parameters were smaller and did not differ systematically by condition or phase: The group mean $b$ parameter estimate was 0.06 (Range: $0.03-0.08$ ) in the proportional phase and 0.06 (Range: $0.02-0.12$ ) in the constant phase. Post hoc tests (Tukey) confirmed that slopes normalized to the long FI duration were significantly flatter in the 8 vs. $32 \mathrm{~s}$ and 2 vs. $32 \mathrm{~s}$ conditions than in all other conditions, and that there were no other significant differences. Although

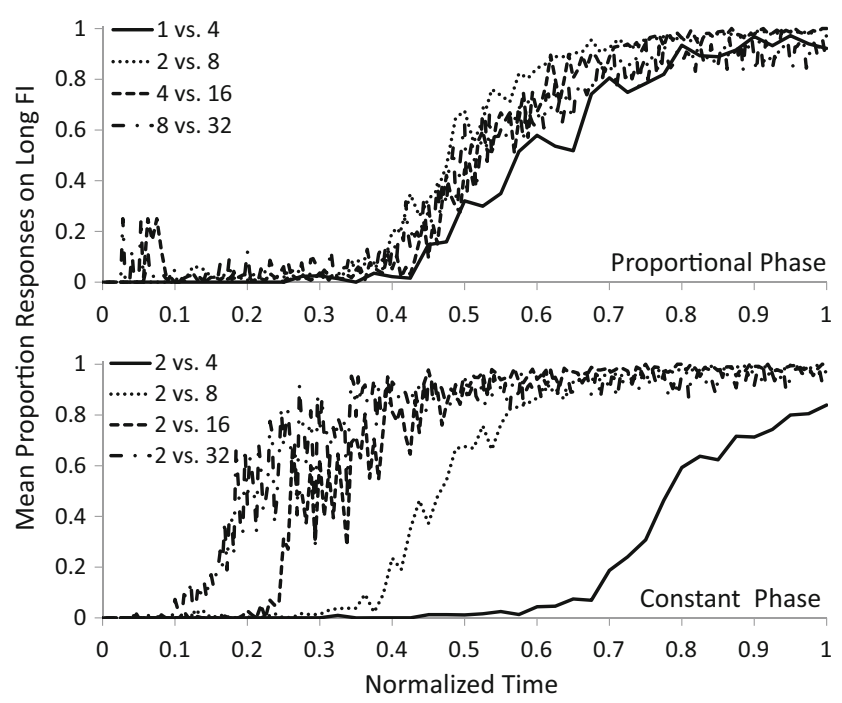

Fig. 2 Group mean psychophysical functions showing the proportion of responding on the long FI in the proportional and constant phases. The 2 vs. $8 \mathrm{~s}$ conditions have been combined in both phases functions were slightly flatter for conditions with the longest FI, there was no evidence of any systematic relation between $b$ values and either the short or long FI duration.

The bisection point $(a)$ parameters for each pigeon in each condition were entered into a $2 \times 4$ repeated-measures ANOVA, with phase and long FI duration as factors. There was a main effect of long FI duration, $F(3,9)=80.10$, $p<.001, \eta_{\mathrm{p}}{ }^{2}=.96$, but no main effect of phase, $F(1,3)=$ $8.93, p=.06, \eta_{\mathrm{p}}{ }^{2}=.75$. The Phase $\times$ Long FI interaction was significant, $F(3,9)=20.37, p<.001, \eta_{\mathrm{p}}{ }^{2}=.87$. Simple effects analysis showed an effect of long FIs in the constant phase, $F(3,9)=52.17, p<.001, \eta_{\mathrm{p}}{ }^{2}=.95$, but not the proportional phase, $F(3,9)=1.69, p=.24, \eta_{\mathrm{p}}{ }^{2}=.36$. Consistent with bisection at the geometric mean, bisection points of psychophysical functions from the constant phase were proportionately earlier for longer long FI durations. Linear trend analysis of individual pigeons' bisection points from the constant phase with condition as factor revealed a monotonic decreasing trend: longer long FI durations in the constant phase produced relatively earlier bisection points, $F(1,3)=153.72$, $p=.001, \eta_{\mathrm{p}}{ }^{2}=.98$. By contrast, relative bisection points were similar in all conditions of the proportional phase: When the short FI was one quarter the duration of the long FI, bisection points were at a mean of $.54(S D=.05)$ of the long FI duration, with little variation across pigeons or conditions.

Figure 3 shows mean bisection points for pigeons in the proportional and constant phases as a function of the geometric mean of the short and long FI in each condition. In both 


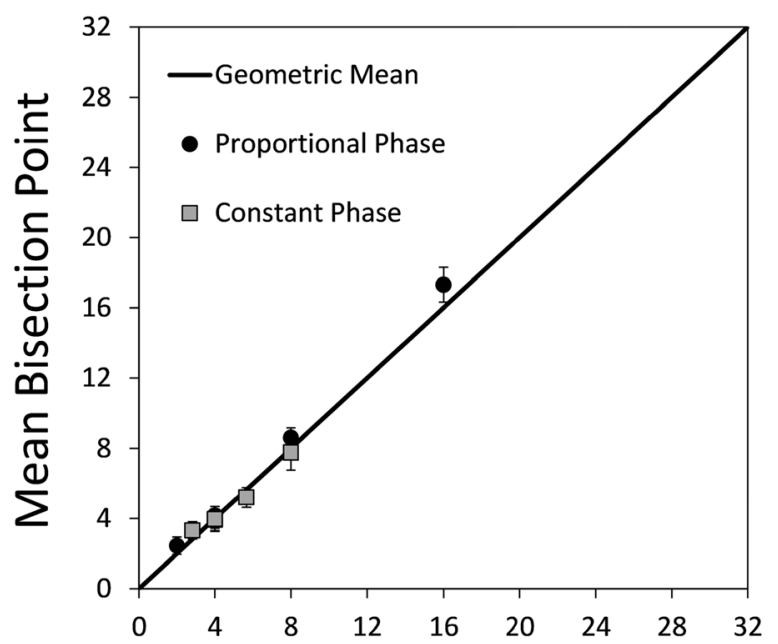

\section{Geometric Mean of Short \& Long FI (s)}

Fig. 3 Mean bisection points for pigeons in the proportional and constant phases. Error bars represent standard deviations. The solid line is the identity line: geometric mean as a function of geometric mean

phases, mean bisection points were near the geometric mean of the short and long FIs, with little intersubject variability.

Long-FI response gradients To evaluate long-FI responding as a function of time in trial, response rates on the long FI were normalized for each pigeon in each condition. Normalized gradients reflect the relative change in response rate over time in the interval by controlling for idiosyncratic differences in absolute response rate. This permits direct comparisons between timing dependent variables across interval durations and subjects. The normalized response rate in each 0.1 -s bin was calculated using Eq. 2:

$X_{\text {inormalized }}=\left(X_{i}-X_{\min }\right) /\left(X_{\max }-X_{\min }\right)$,

where $X_{\mathrm{i}}$ is the response rate in the current bin, $X_{\min }$ is the lowest response rate observed across bins, and $X_{\max }$ is the highest response rate observed across bins. When the response rates in each bin are normalized, a function that ranges from 0 (the normalized minimum) to 1 (the normalized maximum) is obtained across bins.

To characterize the pattern of change in response rate quantitatively, we fit Eq. 1 to the normalized long FI gradients for each pigeon in each condition to maximize variance accounted for (VAC) with a nonlinear optimization algorithm (Microsoft Excel Solver). As before, the $a$ parameter of Eq. 1 determined the center of the function (the time in which the response rate reached half of its maximum), and the $b$ parameter determined the slope of the function at time $t=a$. Smaller estimates of $b$ indicate steeper slopes and therefore more abrupt changes in the response gradients. Table 3 shows parameters obtained from fitting Eq. 1 to response gradients for each pigeon. Mean VAC across pigeons and conditions was $.87(S D=.07$, Range: $0.67-0.97)$.
Across pigeons and conditions, the midpoint ( $a$ parameter in Eq. 1) was 0.75 (Range: 0.60-0.92) of the long FI. Response rate reached half of its maximum when approximately three-quarters of the interval had elapsed. The $a$ parameters for each pigeon in each condition were entered into a $2 \times 4$ repeated-measures ANOVA, with phase (constant or proportional) and long FI duration as factors. The main effect of phase was not statistically significant, $F(1,3)=0.92, p=.41, \eta_{\mathrm{p}}{ }^{2}=.23$, but there was a significant main effect of FI duration, $F(3,9)=12.06, p=.002, \eta_{\mathrm{p}}{ }^{2}=.80$, and a Phase $\times$ FI interaction, $F(3,9)=9.39, p=.004$, $\eta_{\mathrm{p}}{ }^{2}=.76$. The top graph in Fig. 4 shows $95 \%$ confidence limits on $a$ in each condition showing substantial overlap across conditions. In spite of significant effects, differences were not systematic across conditions or between the proportional and constant phases.

The lower graph in Fig. 4 shows 95\% confidence limits on the $b$ parameter in each condition. While there is overlap in the limits, the effect of increasing the long FI is obvious and systematic on the $b$ parameter. In both phases, conditions with longer long FIs produced relatively larger $b$ parameters. To confirm this effect quantitatively, $b$ parameters for each pigeon in each condition were entered into a $2 \times 4$ repeated-measures ANOVA, with phase and long FI duration as factors. There was a main effect of FI duration, $F(3,9)=83.32, p<.001, \eta_{\mathrm{p}}{ }^{2}=.97$. With one exception (Pigeon 501, 2 vs. 8 condition in the proportional phase), $b$ increased monotonically with longer long FIs. The main effect of phase and the Phase $\times$ FI duration interaction were not statistically significant, $F(1,3)=0.92, p=.41, \eta_{\mathrm{p}}{ }^{2}=.23$, and $F(3,9)=2.96, p=.09, \eta_{\mathrm{p}}{ }^{2}=.50$, respectively.

Short-FI response gradients To evaluate short-FI responding as a function of time in trial, response rates on the short FI were normalized for each pigeon in each condition using Eq. 2. To characterize the pattern of change in response rate quantitatively, we fit a Gaussian function to the short FI gradients for each pigeon in each condition to maximize variance accounted for (VAC) with a nonlinear optimization algorithm (Microsoft Excel Solver) using Eq. 3:

$y(t)=e^{-(t-a)^{2} / 2 b^{2}}$,

where $a$ is the time of the peak location, a measure of timing accuracy, and $b$ is the width of the peak distribution, a measure of timing precision. Table 4 shows parameters obtained from fitting Eq. 3 to response gradients from each pigeon. Mean VAC across pigeons and conditions was .76 $(S D=.18$, Range: 0.16-0.96).

Peak locations ( $a$ parameters) normalized to the short FI for each pigeon in each condition were entered into a $2 \times 4$ repeated-measures ANOVA, with phase and long FI duration as factors. The main effect of phase was not statistically significant, $F(1,3)=6.25, p=.09, \eta_{\mathrm{p}}{ }^{2}=.268$, but there was 
Table 3 Ogive model parameter estimates (Eq. 1) for responding on the long FI

\begin{tabular}{|c|c|c|c|c|c|c|c|c|c|c|c|}
\hline \multirow[t]{2}{*}{ Pigeon } & \multirow[t]{2}{*}{ Parameter } & \multicolumn{5}{|c|}{ Proportional Phase } & \multicolumn{5}{|c|}{ Constant Phase } \\
\hline & & 1 vs. 4 & 2 vs. $8(1)$ & 2 vs. 8 (2) & 4 vs. 16 & 8 vs. 32 & 2 vs. 4 & 2 vs. 8 (1) & 2 vs. 8 (2) & 2 vs. 16 & 2 vs. 32 \\
\hline \multirow[t]{4}{*}{501} & Center $(a)$ & 2.73 & 4.80 & 5.97 & 10.85 & 24.27 & 3.32 & 5.32 & 5.28 & 10.16 & 23.82 \\
\hline & Slope $(b)$ & 0.54 & 0.95 & 0.91 & 2.22 & 8.05 & 0.26 & 1.17 & 0.85 & 3.38 & 8.46 \\
\hline & VAC & 0.90 & 0.89 & 0.93 & 0.88 & 0.67 & 0.95 & 0.86 & 0.87 & 0.79 & 0.71 \\
\hline & $\mathrm{CV}$ & 0.20 & 0.20 & 0.15 & 0.20 & 0.33 & 0.08 & 0.22 & 0.16 & 0.33 & 0.36 \\
\hline \multirow[t]{4}{*}{502} & Center $(a)$ & 3.07 & 5.79 & 5.94 & 12.30 & 24.36 & 3.23 & 5.75 & 5.88 & 11.44 & 22.31 \\
\hline & Slope $(b)$ & 0.36 & 1.07 & 0.90 & 2.18 & 7.81 & 0.21 & 0.88 & 1.07 & 2.50 & 6.60 \\
\hline & VAC & 0.96 & 0.87 & 0.93 & 0.89 & 0.74 & 0.97 & 0.90 & 0.94 & 0.91 & 0.87 \\
\hline & $\mathrm{CV}$ & 0.12 & 0.18 & 0.15 & 0.18 & 0.32 & 0.07 & 0.15 & 0.18 & 0.22 & 0.30 \\
\hline \multirow[t]{4}{*}{503} & Center $(a)$ & 2.75 & 5.41 & 5.28 & 11.93 & 25.72 & 3.45 & 6.12 & 4.68 & 10.47 & 24.95 \\
\hline & Slope $(b)$ & 0.46 & 1.00 & 1.07 & 2.91 & 6.03 & 0.30 & 1.07 & 1.12 & 3.14 & 10.76 \\
\hline & VAC & 0.90 & 0.88 & 0.91 & 0.82 & 0.85 & 0.88 & 0.91 & 0.86 & 0.78 & 0.69 \\
\hline & $\mathrm{CV}$ & 0.17 & 0.18 & 0.20 & 0.24 & 0.23 & 0.09 & 0.17 & 0.24 & 0.30 & 0.43 \\
\hline \multirow[t]{4}{*}{504} & Center $(a)$ & 3.29 & 5.99 & 5.94 & 12.52 & 29.36 & 3.52 & 6.18 & 6.66 & 12.40 & 24.65 \\
\hline & Slope $(b)$ & 0.30 & 1.19 & 1.24 & 2.26 & 6.34 & 0.26 & 1.06 & 0.99 & 3.48 & 7.24 \\
\hline & VAC & 0.85 & 0.89 & 0.87 & 0.91 & 0.81 & 0.93 & 0.91 & 0.88 & 0.85 & 0.81 \\
\hline & $\mathrm{CV}$ & 0.09 & 0.20 & 0.21 & 0.18 & 0.22 & 0.07 & 0.17 & 0.15 & 0.28 & 0.29 \\
\hline
\end{tabular}

Note. $\mathrm{VAC}=$ variance accounted for; $\mathrm{CV}=$ coefficient of variation. Paranthetical (1) and (2) in headings respectively refer to the initial 2 vs. 8 condition in a phase and the replication.

a significant main effect of FI duration, $F(3,9)=30.37, p<.001$, $\eta_{\mathrm{p}}{ }^{2}=.92$, and a Phase $\times$ FI interaction, $F(3,9)=16.11, p<.001$,

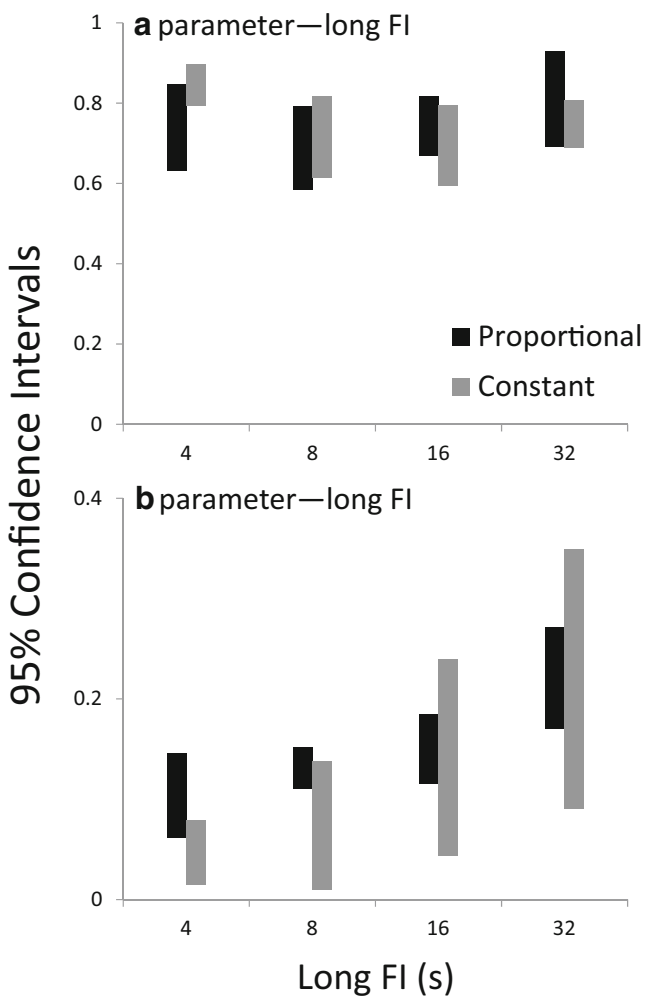

Fig. 4 Ninety-five percent confidence intervals for the $a$ and $b$ parameters from fitting Eq. 1 to response gradients for the long FI in the proportional and constant phases $\eta_{\mathrm{p}}{ }^{2}=.84$. The top graph in Fig. 5 shows $95 \%$ confidence limits on $a$ in each condition. In all but one condition, local response rate on the short FI key was greatest at the approximate time of food delivery on short FI trials. The peak location for the $1 \mathrm{vs} .4 \mathrm{~s}$ condition was relatively later in the trial. It may be that at the start of a trial, the pigeons responded in a bout (Shull, Gaynor, \& Grimes, 2001) on the short FI before switching to the long FI (i.e., pigeons were not necessarily timing the short FI in the 1 vs. $4 \mathrm{~s}$ condition). It is also possible gradients are shifted rightward because pigeons were still consuming the reinforcer from the previous trial, and this delayed the onset of pecking to the FI 1 s. Post hoc tests (Tukey) confirmed that peak location was significantly later in the 1 vs. $4 \mathrm{~s}$ condition than in all other conditions, and that there were no other significant differences.

The bottom graph in Fig. 5 shows $95 \%$ confidence limits on the standard deviation of the short-FI response gradient (the $b$ parameter in Eq. 3) in each condition. Standard deviation (b) parameters for each pigeon in each condition were entered into a $2 \times 4$ repeated-measures ANOVA, with phase and long FI duration as factors. There was a main effect of long FI duration, $F(3,9)=3.98, p=.047, \eta_{\mathrm{p}}{ }^{2}=.57$ : Longer long FI durations increased the relative width of the short FI response gradient, an indication of general interference by some aspect of the long FI on short FI timing. There was no main effect of phase, $F(1,3)=$ $5.70, p=.10, \eta_{\mathrm{p}}{ }^{2}=.66$, or Phase $\times$ Long FI interaction, $F(3,9)$ $=1.51, p=.28, \eta_{\mathrm{p}}{ }^{2}=.34$; however, an analysis of simple effects showed significantly wider relative widths at longer long FIs in the proportional phase, $F(3,9)=5.14, p<.001, \eta_{\mathrm{p}}{ }^{2}=.68$, but 
Table 4 Gaussian model parameter estimates (Eq. 3) for responding on the short FI In the table note, please explain the meaning of parenthetical (1) and (2).Thanks, we will add the statement:. Paranthetical (1) and (2) in headings respectively refer to the initial 2 vs. 8 condition in a phase and the replication.

\begin{tabular}{|c|c|c|c|c|c|c|c|c|c|c|c|}
\hline \multirow[t]{2}{*}{ Pigeon } & \multirow[t]{2}{*}{ Parameter } & \multicolumn{5}{|c|}{ Proportional Phase } & \multicolumn{5}{|c|}{ Constant Phase } \\
\hline & & 1 vs. 4 & 2 vs. 8 (1) & 2 vs. 8 (2) & 4 vs. 16 & 8 vs. 32 & 2 vs. 4 & 2 vs. 8 (1) & 2 vs. 8 (2) & 2 vs. 16 & 2 vs. 32 \\
\hline \multirow[t]{4}{*}{501} & Peak $(a)$ & 1.42 & 1.98 & 2.38 & 4.23 & 8.19 & 1.97 & 2.01 & 1.96 & 2.08 & 2.23 \\
\hline & Width $(b)$ & 0.21 & 0.46 & 0.60 & 1.32 & 2.50 & 0.27 & 0.37 & 0.31 & 0.40 & 0.42 \\
\hline & VAC & 0.84 & 0.82 & 0.82 & 0.81 & 0.55 & 0.83 & 0.94 & 0.94 & 0.91 & 0.93 \\
\hline & $\mathrm{CV}$ & 0.15 & 0.23 & 0.25 & 0.31 & 0.31 & 0.14 & 0.18 & 0.16 & 0.19 & 0.19 \\
\hline \multirow[t]{4}{*}{502} & Peak $(a)$ & 1.52 & 2.05 & 2.13 & 4.44 & 7.65 & 2.00 & 2.12 & 2.08 & 2.13 & 2.19 \\
\hline & Width $(b)$ & 0.33 & 0.40 & 0.40 & 1.11 & 2.60 & 0.36 & 0.38 & 0.33 & 0.32 & 0.34 \\
\hline & VAC & 0.71 & 0.84 & 0.81 & 0.80 & 0.66 & 0.88 & 0.95 & 0.96 & 0.89 & 0.86 \\
\hline & $\mathrm{CV}$ & 0.22 & 0.20 & 0.19 & 0.25 & 0.34 & 0.18 & 0.18 & 0.16 & 0.15 & 0.16 \\
\hline \multirow[t]{4}{*}{503} & Peak $(a)$ & 1.21 & 1.99 & 1.80 & 3.72 & 7.78 & 2.16 & 2.11 & 1.77 & 2.01 & 2.21 \\
\hline & Width $(b)$ & 0.22 & 0.52 & 0.47 & 1.05 & 2.19 & 0.53 & 0.49 & 0.44 & 0.58 & 0.60 \\
\hline & VAC & 0.68 & 0.69 & 0.79 & 0.84 & 0.56 & 0.16 & 0.76 & 0.75 & 0.85 & 0.74 \\
\hline & $\mathrm{CV}$ & 0.18 & 0.26 & 0.26 & 0.28 & 0.28 & 0.25 & 0.23 & 0.25 & 0.29 & 0.27 \\
\hline \multirow[t]{4}{*}{504} & Peak $(a)$ & 1.48 & 1.98 & 2.00 & 4.05 & 9.26 & 2.10 & 2.02 & 1.98 & 2.26 & 2.19 \\
\hline & Width $(b)$ & 0.23 & 0.27 & 0.35 & 1.12 & 4.12 & 0.38 & 0.33 & 0.25 & 0.39 & 0.37 \\
\hline & VAC & 0.21 & 0.77 & 0.81 & 0.47 & 0.56 & 0.78 & 0.84 & 0.70 & 0.93 & 0.77 \\
\hline & $\mathrm{CV}$ & 0.16 & 0.14 & 0.18 & 0.28 & 0.44 & 0.18 & 0.16 & 0.13 & 0.17 & 0.17 \\
\hline
\end{tabular}

Note. VAC $=$ variance accounted for; $\mathrm{CV}=$ coefficient of variation. Paranthetical (1) and (2) in headings respectively refer to the initial 2 vs. 8 condition in a phase and the replication

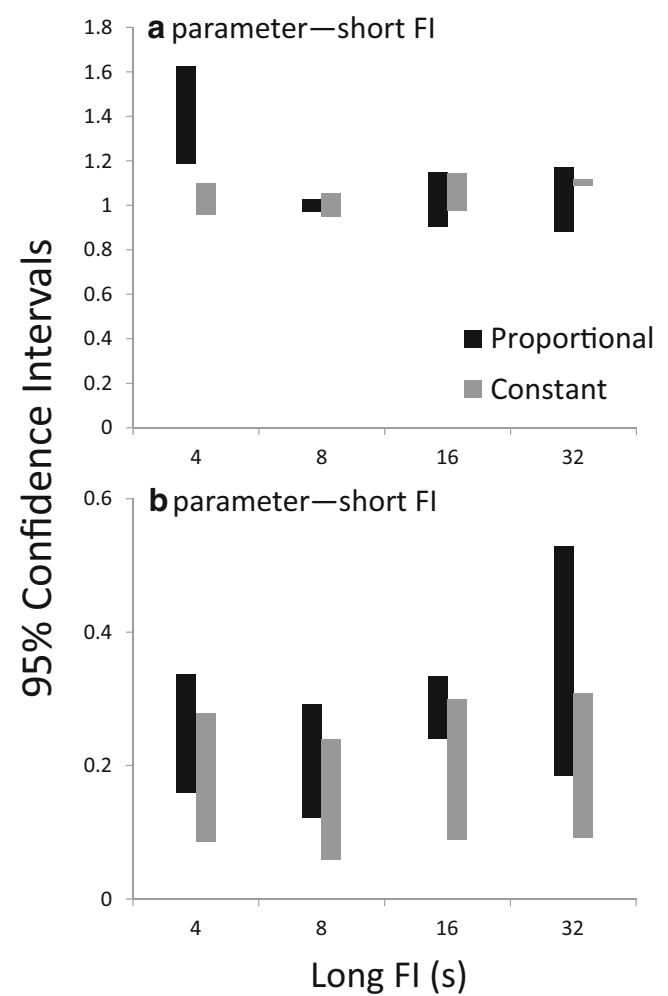

Fig. 5 Ninety-five percent confidence intervals for the $a$ and $b$ parameters from fitting Eq. 3 to response gradients for the short FI in the proportional and constant phases not the constant phase, $F(3,9)=0.33, p=.60, \eta_{\mathrm{p}}{ }^{2}=.17$. Simple effects suggest that widths of short FI gradients were determined in part by the short:long ratio. At differences greater than 1:4 between the long and short FI, respectively, the long FI interfered less, if at all, with timing of the short FI.

Coefficients of variation The standard deviation parameter ( $b$; Gaussian width) divided by the peak location parameter (a) of the Gaussian in Eq. 3 yields an index of normalized timing precision for the short-FI response gradients that has been widely used in studies investigating timing in the peak procedure. The slope parameter $(b)$ divided by the center or bisection point parameter $(a)$ of the ogive function, Eq. 1, yields a similar index of normalized timing precision for the long-FI gradients and the psychophysical functions that has been validated by previous research (Guilhardi \& Church, 2005). These are CVs for each of the timing measures.

Figure 6 shows group mean CVs from all phases and conditions for the ogive fits to psychophysical functions depicting the proportion of responding on the long FI (top), ogive fits to the long-FI gradients (middle), and the Gaussian fits to the short-FI gradients (bottom). If scalar variance held in this procedure, $\mathrm{CV}$ s for each function would be the same across conditions and phases. That is, the lines of best fit for both phases in each panel of Fig. 6 would have slopes of zero. Only CVs for the Gaussian fits to the short-FI response gradients from 

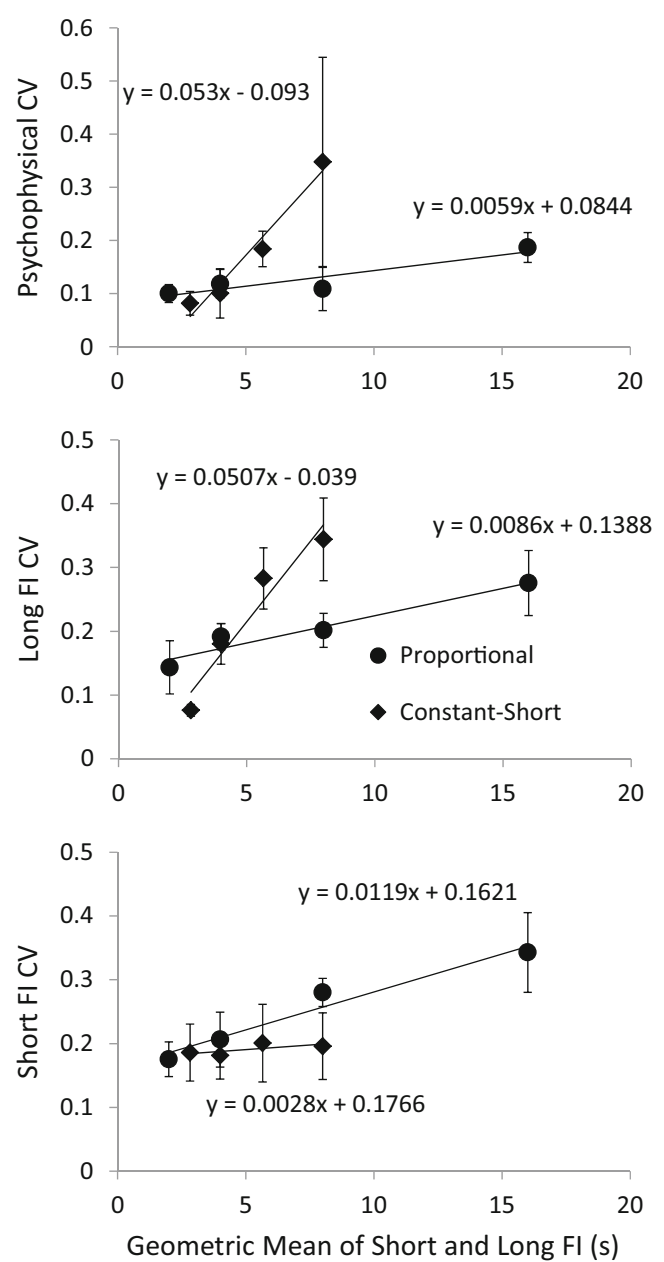

Fig. 6 Mean coefficients of variation (CVs) from pigeons in the proportional (circles) and constant (diamonds) phases as a function of the geometric mean of the short and long FI. The top panel shows mean CVs for psychophysical ogive gradients based on parameter estimates from Eq. 1, the middle panel shows mean CVs for long FI ogive gradients based on parameter estimates from Eq. 1, and the bottom panel shows mean CVs for short FI Gaussian gradients based on parameter estimates from Eq. 3. Error bars represent standard deviations

the constant phase (diamonds, bottom panel) were constant as the long FI increased. Pigeons timed the 2-s short FI with equivalent precision regardless of the duration of the corresponding long FI. Short-FI CVs in the proportional phase increased systematically as the intervals increased, as did CVs in both phases for the long FI and psychophysical functions. For the long FI and psychophysical functions, slopes from regressing CVs on the geometric mean of the long and short FIs were higher in the constant phase than in the proportional phase, indicating that the difference in relative variability as a function of FI duration was greater in the constant phase than the proportional phase. These deviations are inconsistent with the scalar property of timing and suggest simultaneous timing of the two FIs interfered with timing precision.

\section{Discussion}

In this experiment, four pigeons were exposed to an FI temporal bisection task in two phases: one in which the short and long FIs changed proportionally and one in which the short FI was constant but the long FI changed. Similar to Platt and Davis's (1983) experiments, the psychophysical functions characterizing the proportion of long responses as a function of time were ogival with bisection points near the geometric mean of the short and long intervals. This finding is consistent with temporal bisection task research using a similar freeoperant procedure (Fetterman \& Killeen, 1995; Platt \& Davis, 1983) and using the more widespread version of the temporal bisection task in which interval durations are categorized as short or long by subjects in a discrete-trial fashion (e.g., Church \& Deluty, 1977). In addition, response gradients for the long FI key were well characterized by ogive functions, and, when the long FI was active, response gradients for the short FI key were well-characterized by Gaussian functions with peak locations at the short FI duration. In this way, the temporal patterns of pecking the long and short FI keys were comparable to patterns of responding in FI schedules of reinforcement (Dews, 1970) and of responding in no-food trials of peak procedures (Roberts, 1981), respectively.

In addition, our results suggest that the two FIs in this procedure are timed simultaneously, but that the timing of one FI interfered with timing the other. This produced deviations from scalar variability and differences when comparing dependent measures in this task to similar dependent measures from other timing procedures - two issues we will now explore more thoroughly for each of the dependent measures separately.

Psychophysical functions The point in the interval when responding was most likely to switch from the short FI key to the long FI key is the bisection point, the center of the psychophysical function. Consistent with typical performance in free-operant FI bisection (Platt \& Davis, 1983) and discretetrial temporal bisection tasks (e.g., Church \& Deluty, 1977), bisection points were near the geometric mean of the short and long FIs in all conditions.

Interestingly, Platt and Davis (1983) reported that at short:long FI duration ratios greater than 1:4, bisection points no longer occurred consistently at the geometric mean of the two intervals. They hypothesized that when the ratios were greater than 1:4, the intervals exerted independent control on behavior; in other words, the pigeons were timing the intervals separately and consecutively without interference. We employed ratios up to 1:16 and observed bisection points at the geometric means of the two intervals, suggesting simultaneous control by the two intervals at ratios considerably larger than 1:4. As the ratio increased in the constant phase though, 
the long FI duration interfered with timing the short FI less, as evidenced by constant CVs for timing on the short FI.

We observed higher psychophysical function $\mathrm{CV}$ s for conditions with longer geometric means. This violation of scalar variance has been reported in mice on a task very similar to the one used here (Balci et al., 2008). There are also reports of scalar variance being violated in a similar manner in temporal bisection tasks conducted with humans. In these studies, CVs increased slightly as the absolute durations being timed got longer (Allan \& Gibbon, 1991; Wearden, 1991, 1995; Wearden \& Ferrara, 1995), or the short:long ratio decreased (Kopec \& Brody, 2010). Violations of scalar variance have also been documented in rats (e.g., Crystal, 1999, 2001). Together, these findings suggest increased interference in timing processes as multiple simultaneous intervals to be timed increase.

Long-FI response gradients There are two characteristic properties of responding in simple FI schedules: scalar timing and scalar variance (Lejeune \& Wearden, 2006). With scalar timing, the center of the response gradient is proportional to the duration of the interval. In single FI schedules, the point when responding was most likely to switch from a low to a high rate is characteristically approximately two-thirds of the FI duration (Schneider, 1969). Ogive centers presently were approximately three-quarters of long FI duration. Scalar timing was observed, but consistently later in the long FI interval than in simple FI schedules, suggesting that responding on the short FI key delayed the onset of responding on the long FI key.

Scalar variance is the requirement that the relative precision of the response gradient is proportional to the duration of the interval, which yields a constant CV (Gibbon, 1977; Guilhardi \& Church, 2005). We did not observe scalar variance in the long FI response gradients. Relative variability was greater in conditions with longer FIs, indicating that the short FI interfered with long FI timing by reducing response gradient precision. The difference was more pronounced in the constant phase for all pigeons, which suggests the amount of disruption was partially determined by the long FI:short FI ratio. Altogether, these findings mean that long FI response gradients obtained from this procedure are similar but not equivalent to response gradients obtained from single FI procedures.

Short-FI response gradients In peak procedures, the response gradient on no-food intervals is a Gaussian distribution, with a peak location approximately equal to the duration of the reinforced intervals and a standard deviation (width) that is proportional to the mean. In the present experiment, response gradients peaked at the approximate time of the programmed short FI. With the exception of the FI 1-s short FI, peak locations were all close to the short FI duration. However, we did not observe scalar variance in the proportional phase: CVs were greater for conditions with longer FIs. By contrast, short FI CVs were constant in the constant phase when the short FI was always $2 \mathrm{~s}$. Together, the systematic differences in proportional-phase CVs and equal CVs in the constant phase are indications that the presence of the long FI in this procedure may have disrupted short-FI timing by reducing the precision of the response gradient. These results are consistent with the possibility that pigeons learned to ignore the long FI for the first $2 \mathrm{~s}$ of all intervals of the constant phase - this seems especially probable when the long FI was greater than four times the short (Platt \& Davis, 1983), though systematically increasing bisection points and long FI CVs as the long FI increased in the constant phase indicate continued simultaneous control and interference of timing the two FIs in the constant phase.

Coefficients of variation Variance was not scalar for either short or long FI response gradients or for the psychophysical function, indicating that simultaneous timing of the short and long FI schedules may have interfered with timing precision. Relative variability was greater in conditions with longer geometric means, which suggests that each schedule interfered with timing precision in the other and that longer schedules (for either short or long FI) incited relatively more interference. However, short-FI Gaussian CVs were similar across pigeons and conditions in the constant phase, which suggests that neither absolute FI nor short:long ratio can entirely account for violations of scalar variance. The simplest way to consider this is that timing two FIs simultaneously resulted in greater relative variation as the FIs got longer - a violation of scalar variance consistent with previous research employing a similar procedure (Balci et al., 2008).

Together, previous and present findings employing the Platt and Davis (1983) FI temporal bisection task are promising and suggest that the procedure is a useful tool for evaluating a wide range of timing-related independent variables in behavioral and neurological research. Combining spatial, temporal, and decision-making variables, the procedure may better emulate real-life types of scenarios. The current study highlights potential use of multiple dependent variables derived from the task, characterizes them, and suggests that independent variable manipulations may affect these dependent measures differently.

Our results suggest that researchers employing this task should consider employing multiple dependent measures instead of just the bisection/switch points because changes in different dependent measures may suggest fundamentally different assumptions about their results. For example, a procedure designed to assess the effects of reducing reinforcer efficacy on timing (e.g., prefeeding) may result in later bisection points. This might indicate a subjective slowing of time (i.e. slower "clock" speed; Gibbon, 1977), a modulation of the threshold to switch from the short to the long FI (e.g., Daniels, et al., 2015a), or both. Only by assessing the relative effects of other dependent measures might this be teased out. For example, a 
shift in peak times on the short FI response gradients and shifts in bisection points indicate a shift in the subjective speed with which time is passing; however, a shift in bisection points alone indicates a modulation in the threshold to switch to the long FI but not a change in the subjective speed of time.

As the task is increasingly used in animal models, it will also be important to assess the generality of the procedure to human subjects (see Daniels, et al., 2015a; Fox, Prue, \& Kyonka, 2016). Now that nonhuman behavior in the task is well characterized, cross-species generalizations can be investigated across multiple dependent measures.

\section{References}

Allan, L. G., \& Gibbon, J. (1991). Human bisection at the geometric mean. Learning and Motivation, 22, 39-58.

Balci, F., Freestone, D., \& Gallistel, C. R. (2009). Risk assessment in man and mouse. Proceedings of the National Academy of Sciences, 106, 2459-2463.

Balci, F., Papchristos, E. B., Gallistel, C. R., Brunner, D., Gibson, J., \& Shumyatsky, G. P. (2008). Interval timing in genetically modified mice: A simple paradigm. Genes, Brain and Behavior, 7, 373-384.

Bizo, L. A., \& White, G. (1994). Pacemaker rate in the behavioral theory of timing. Journal of Experimental Psychology: Animal Behavior Processes, 20, 308-321.

Bizo, L. A., \& White, G. (1995). Reinforcement context and pacemaker rate in the behavioral theory of timing. Animal Learning \& Behavior, 23, 376-382.

Catania, A. C. (1970). Reinforcement schedules and pscyho-physical judgments: A study of some temporal properties of behavior. In W. N. Schoenfeld (Ed.), The theory of reinforcement schedules (pp. ). New York: Appleton-Century-Croft.

Cheung, T. H. C., Bezzina, G., Hampson, C. L., Body, S., Fone, K. C. F., Bradshaw, C. M., \& Szabadi, E. (2007). Evidence for the sensitivity of operant timing behaviour to stimulation of D1 dopamine receptors. Psychopharmacology, 195, 213-222.

Church, R. M., \& Deluty, M. Z. (1977). Bisection of temporal intervals. Journal of Experimental Psychology: Animal Behavior Processes, 3, 216-228

Cordes, S., \& Meck, W. H. (2014). Ordinal judgments in the rat: An understanding of longer and shorter for suprasecond, but not subsecond, durations. Journal of Experimental Psychology: General., 143, 710-720.

Crystal, J. D. (1999). Systematic nonlinearities in the perception of temporal intervals. Journal of Experimental Psychology: Animal Behavior Processes, 25, 3-17.

Crystal, J. D. (2001). Nonlinear time perception. Behavioural Processes, $55,35-49$.

Daniels, C. W., Fox, A. E., Kyonka, E. G. E. \& Sanabria, F. (2015a) Biasing temporal judgments in rats, pigeons, and humans. International Journal of Comparative Psychology, 28. uclapsych ijcp 27337. Retrieved from: http://escholarship.org/uc/ item/50n6389s.

Daniels, C. W., Watterson, E., Garcia, R., Mazur, G. J., Brackney, R. J., \& Sanabria, F. (2015b). Revisiting the effect of nicotine on interval timing. Behavioural Brain Research, 283, 238-250.

Dews, P. B. (1970). The theory of fixed-interval responding. In W. N. Schoenfeld (Ed.), The theory of reinforcement schedules (pp. 4362). New York: Appleton-Century-Crofts.
Fetterman, J. G., \& Killeen, P. (1995). Categorical scaling of time: Implications for clock-counter models. Journal of Experimental Psychology: Animal Behavior Processes, 21, 43-63.

Fox, A. E., Prue, K. E., \& Kyonka, E. G. E. (2016). Human timing in a fixed-interval temporal bisection procedure. Manuscript submitted for publication.

Gallistel, C. R., Tucci, V., Nolan, P. M., Schachner, M., Jakovcevski, I., Kheifets, A., \& Barboza, L. (2014). Cognitive assessment of mouse strains heterozygous for cell-adhesion genes reveals strain-specific alterations in timing. Philosophical Transactions of the Royal Society, 369. doi:10.1098/rstb.2012.0464

Gibbon, J. (1977). Scalar expectancy theory and Weber's law in animal timing. Psychological Review, 84, 279-325.

Guilhardi, P., \& Church, R. M. (2005). Dynamics of temporal discrimination. Learning \& Behavior, 33, 399-416.

Kopec, C. D., \& Brody, C. D. (2010). Human performance on the temporal bisection task. Brain and Cognition, 74, 262-272.

Lejeune, H., \& Wearden, J. H. (2006). Scalar properties in animal timing: Conformity and violations. Quarterly Journal of Experimental Psychology, 59, 1875-1908.

Machado, A., \& Pata, P. (2005). Testing the scalar expectancy theory (SET) and the learning-to-time model (LeT) in a double bisection task. Learning \& Behavior, 33, 111-222.

Maggi, S., Garbugino, L., Heise, I., Nieus, T., Balci, F., Wells, S., . . Tucci, V. (2014). A cross-laboratory investigation of timing endophenotypes in mouse behavior. Timing \& Time Perception, 2, 35-50.

Pinheiro de Carvalho, M., Machado, A., \& Tonneau, F. (2016). Learning in the temporal bisection task: Relative or absolute? Journal of Experimental Psychology: Animal Learning and Cognition, 42, 67-81.

Platt, J. R., \& Davis, E. R. (1983). Bisection of temporal intervals by pigeons. Journal of Experimental Psychology: Animal Behavior Processes, 9, 160-170.

Roberts, S. (1981). Isolation of an internal clock. Journal of Experimental Psychology: Animal Behavior Processes, 7, 242-268.

Sargisson, R. J., Lockhart, R. A., McEwan, J. S., \& Bizo, L. A. (2016). Demonstration of the scalar property of timing with possums (Trichosurus vulpecula). Journal of Comparative Psychology. doi: 10.1037/com0000022

Schneider, B. A. (1969). A two-state analysis of fixed-interval responding in the pigeon. Journal of the Experimental Analysis of Behavior, 12, 677-687. doi:10.1901/jeab.1969.12-677.

Shull, R. L., Gaynor, S. T., \& Grimes, J. A. (2001). Response rate viewed as engagement bouts: Effects of relative reinforcement and schedule type. Journal of the Experimental Analysis of Behavior, 75, 247-274.

Staddon, J. E. R., \& Innis, N. K. (1969). Reinforcement omission on fixed-interval schedules. Journal of the Experimental Analysis of Behavior, 12, 689-700.

Stubbs, D. A. (1980). Temporal discrimination and a free-operant psychophysical procedure. Journal of the Experimental Analysis of Behavior, 33, 167-185.

Tosun, T., Gur, E., \& Balci, F. (2016). Mice plan decision strategies based on previously learned time intervals, locations, and probabilities. Proceedings of the National Academy of Sciences, 113, 787-792.

Wearden, J. H. (1991). Human performance on an analogue of an interval bisection task. Quarterly Journal of Experimental Psychology, 43B, 59-81.

Wearden, J. H. (1995). Categorical scaling of stimulus duration by humans. Journal of Experimental Psychology: Animal Behavior Processes, 21, 318-330.

Wearden, J. H., \& Ferrara, A. (1995). Stimulus spacing effects in temporal bisection by humans. Quarterly Journal of Experimental Psychology, 48B, 289-310.

Wilson, A. G., Matell, M. S., \& Crystal, J. D. (2015). The influence of multiple temporal memories in the peak-interval procedure. Learning \& Behavior, 43, 153-162. doi:10.3758/ s13420-015-0169-y 